Bangladesh J. Sci. Ind. Res. 42(3), 249-256, 2007

\title{
A Fast Procedure for Transmission Loss Allocation of Power Systems by Using DC-OPF
}

\author{
M. H. Kabir \\ Department of Computer Science and Telecommunication Engineering, \\ Noakhali Science and Technology University, Noakhali 3802, Bangladesh
}

\begin{abstract}
Deregulation of power industry has made us think over the transmission loss allocation to network users and to various interconnected transactions including bilateral one. It is an important issue to be solved exactly. Because of the non-linear nature of power flow and power loss, it is very difficult to segregate and to allocate losses among the participants properly. This paper presents an instantaneous loss allocation algorithm including DC-Optimal Power Flow. Using Incremental Transmission Loss (ITLs) factors and the power outputs of generators, we have calculated preliminary loss to each generator. Calculating loss allocation rates from the preliminary losses, the final loss allocation has been done (according to loss allocation rate) to meet the total loss calculated by DC-OPF. The effectiveness of this procedure has been studied for a small model power system and also for the IEEE-118-bus system.
\end{abstract}

Keywords: Transmission loss allocation, DC optimal power flow, Deregulated power market, Bilateral transactions.

\section{Introduction}

The market driven transactions, under open access environment, have become the new independent decision variables defining the behavior of the power system (Yamashiro, 1977 and Chiba, et al. 2003). Taking into account the real impact of every transaction on the transmission system (Willis, et al. 1996), one of the key issues in the restructured environment that refers to the way the cost of transmission services is satisfactory loss allocation among all involved parties as accurately as possible. Realizing the impact of bilateral transactions on system losses is important to allocate the corresponding loss component to each individual transaction. The bilateral transactions are usually longterm agreements determined through individual negotiations between a buyer and a seller. The price agreed to in a bilateral exchange is based on market forces. The bilateral market needs, in order to improve economic efficiency, the knowledge of the transmission losses associated with each proposed transaction. This knowledge permits both buyer and seller to incorporate the level and cost of losses into their negotiations. Therefore, this 
paper presents a simple approach for allocating losses to the generators in a network including bilateral transactions. A widely accepted approach to allocate losses to generators is based on incremental loss factors those are related to angle of node voltages, line parameters and output power of the generators.

\section{Mathematical Formulation}

In this paper, the minimum load level of the system has been calculated from Eq. (1).

$$
\text { Load }=P D_{i j}+\Sigma(\text { Pmin })_{n}
$$

Here, the minimum limits of the generators (Pmin) $)_{n}$ have excluded bilateral generator $P_{i}$ which is under bilateral contract with load bus $D_{j}$ as denoted by $P D_{i j}$.

The partial derivative of total loss with respect to phase angle $\mathrm{q}$ of a node voltage (Yamashiro, 1977) assumed to be 1 p.u. in every node is

$$
\frac{\partial L}{\partial \theta_{n}}=-2 \sum_{k=1}^{N} G_{n k} \operatorname{Sin}\left(\theta_{n}-\theta_{k}\right)
$$

where $G_{n k}$ is the real part of transfer admittance. $\theta_{n}$ and $\theta_{k}$ are phase angles of voltages at bus $n$ and bus $k$ respectively in a system of

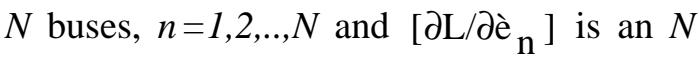
dimensional row vector. We know, there is a close relationship between node power injection at bus n, node voltage angle and susceptance matrix of the network.
Therefore, the ITL (Yamashiro, 1977) has been calculated as the following Eq. (3).

$$
\frac{\partial L}{\partial P_{n}}=\frac{\partial L}{\partial \theta_{n}} \times B^{-1}
$$

where $B$ is an $(N \times N)$ susceptance matrix. From generated power $P_{n}, P_{i}$ and by using Eq. (3) we can calculate the Preliminary Loss $\left(L_{p}\right)$ allocated to generator $n$ and $i$ as

$$
\begin{aligned}
& L_{p n}=P_{n} \times \frac{\partial L}{\partial P_{n}} \\
& L_{p i}=P_{i} \times \frac{\partial L}{\partial P}
\end{aligned}
$$

Where $\left[\partial \mathrm{L} / \partial \mathrm{è}_{\mathrm{n}}\right]$ is the sensitivity of the system losses with respect to injection at bus $n$ and so is $\left[\partial \mathrm{L} / \partial \mathrm{è}_{\mathrm{i}}\right]$ at bus $i$. These are the well-known ITLs (Elgerd, 1982). The sum of preliminary losses, $\left(L_{p i}+\Sigma L_{p n}\right)$, is not equal to the total loss $\boldsymbol{L}$ calculated by DC-OPF. Assuming that the loss allocation can be done according to the proportion of preliminary losses, the Loss Allocation Rates $\left(R_{n}\right.$ and $\left.R_{i}\right)$ have been calculated as

$$
\begin{gathered}
R_{n}=L_{p n} /\left(L_{p i}+\Sigma L_{p n}\right) \\
\text { and } R_{i}=L_{p i} /\left(L_{p i}+\Sigma L_{p n}\right)
\end{gathered}
$$

for generators $n$ and $i$ respectively. Therefore, at a specific load level, the final loss allocation to generator $n$ becomes

$$
\mathrm{L}_{\mathrm{n}}=\mathrm{R}_{\mathrm{n}} \times \mathrm{L}
$$


and that of to generator i (under bilateral contracts) becomes

$$
\mathrm{L}_{\mathrm{i}}=\mathrm{R}_{\mathrm{i}} \times \mathrm{L}
$$

Now, the total of allocated losses is equal to the total loss calculated by DC-OPF of the network. i.e. $L_{i}+\Sigma L_{n}=L$.

\section{Algorithm for transmission loss allocation}

To allocate transmission losses to the generators in a network, we need to estimate the transmission loss allocation rates by Eq. (6) and Eq. (7) those have been calculated from the preliminary losses found from Eq. (4) and Eq. (5). Using them, we can effectively estimate the loss allocation to the generators. In this paper, we have chosen the minimum level of the system operation with a load as in Eq. (1) that depends on bilateral power and the minimum limits of the generators except that of bilateral generator.

Using the well-known lambda search technique, power output of each generator has been determined corresponding to total load and loss excluding bilaterally contracted power. DC-OPF has been used for the calculation of total loss $L$, phase angle $\theta$ of voltage of every node in the system. Hence, ITL and penalty factors have been calculated. The proposed algorithm shown in Fig.1 has been described below.

Step1: Set total loss $=0$, penalty factor $=1$ etc. Specify bilateral contracts between generator $P_{i}$ and load bus $D_{j}$ and also set a specific load level depending on bilateral power $P_{i}$. Minimum load level is found from Eq. (1).

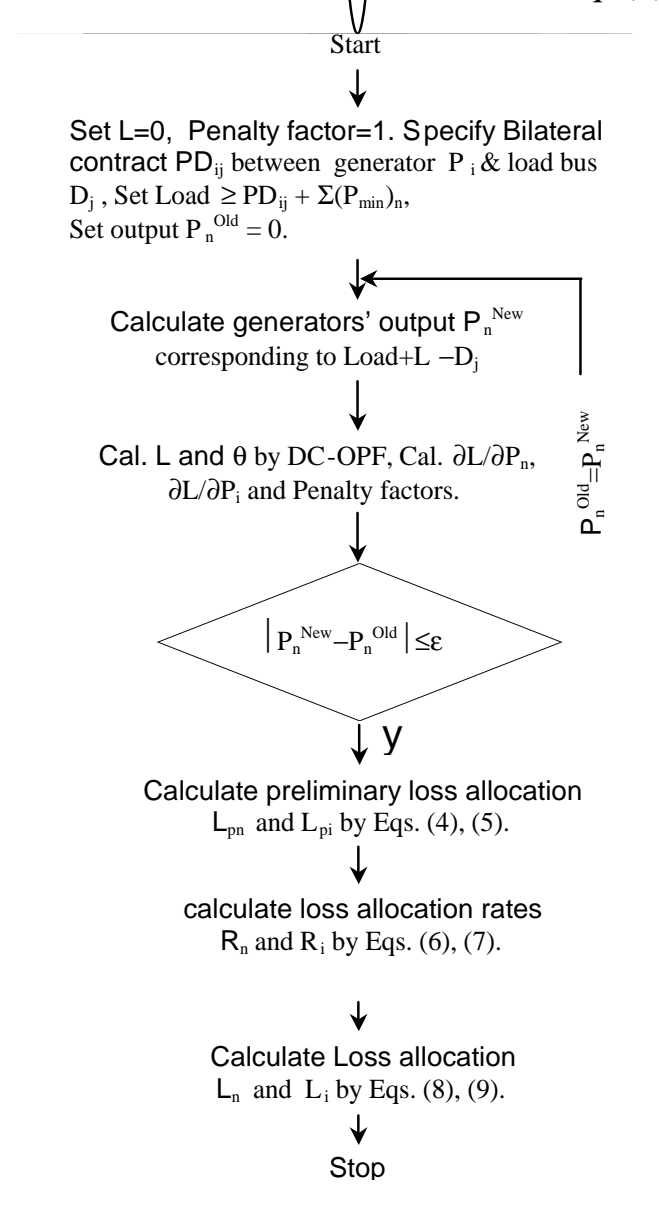

Fig. 1. Flowchart for loss allocation procedure.

Step2: Outputs of the generators are calculated in the mode of economic load dispatch using lambda search technique. Total power $P=$ load + loss $-D_{j}$.

Step3: Applying DC-OPF, the total transmission loss, angle of every node voltage, ITLs $\partial \mathrm{L} / \partial \mathrm{P}_{\mathrm{n}}, \partial \mathrm{L} / \partial \mathrm{P}_{\mathrm{i}}$ and penalty factors of the generators are obtained. 
Step4: The output power $P_{n}$ of the generator $\mathrm{n}$ and the preliminary loss allocation $L_{p n}$ and $L_{p i}$ are calculated.

Step5: The Loss allocation rates are calculated from the preliminary losses. Loss allocation $L_{n}$ has been calculated for generator $n$ and $L_{i}$ for bilateral generator $i$.

\section{Simulation Results}

To represent the effectiveness of our proposed algorithm as shown in Fig.1, the simulation results have been calculated for the following model power system (Fig.2).

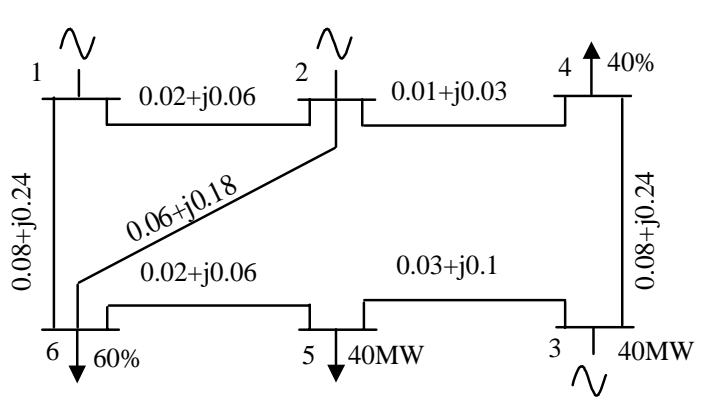

Fig. 2. Model power system

Table I. Characteristic constants of thermal units

\begin{tabular}{c|c|c|c|c|c}
\hline Unit & \multicolumn{4}{|c|}{ Fuel cost curve constants } & \multicolumn{3}{|c}{$\begin{array}{c}\text { Output power (MW) } \\
\text { no. }\end{array}$} & A & B & C & Min. & Max. \\
\hline 1 & 15.3 & 1.17 & 0.00145 & 80 & 225 \\
2 & 13.7 & 1.30 & 0.00163 & 40 & 175 \\
3 & 10.3 & 1.48 & 0.00226 & 25 & 75 \\
\hline
\end{tabular}

Here fuel cost function is $F(P)=$ $A+B P+C P^{2}$. We have assumed that generator 3 is under bilateral contract with the load bus 5. Bilaterally contracted power is $40 \mathrm{MW}$ i.e. $\mathrm{PD}_{35}=40 \mathrm{MW}$.

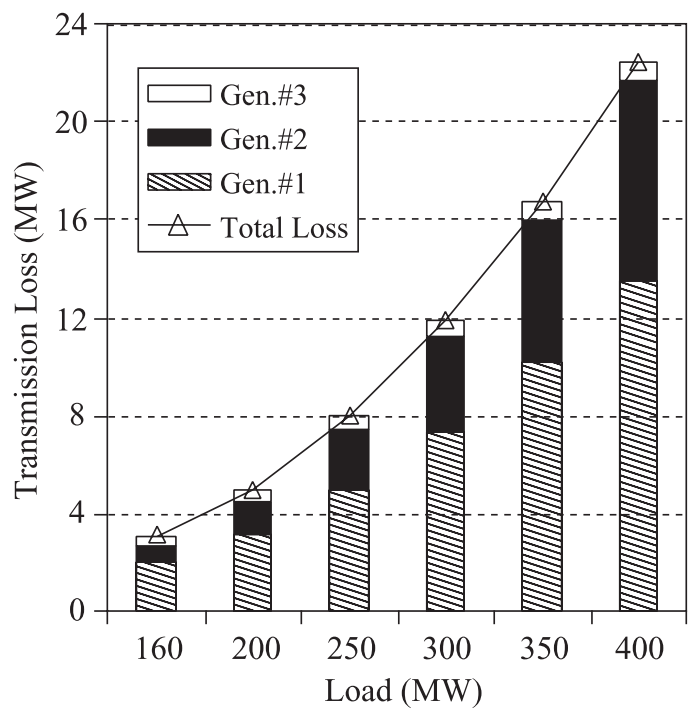

Fig. 3. Loss allocation at several load levels

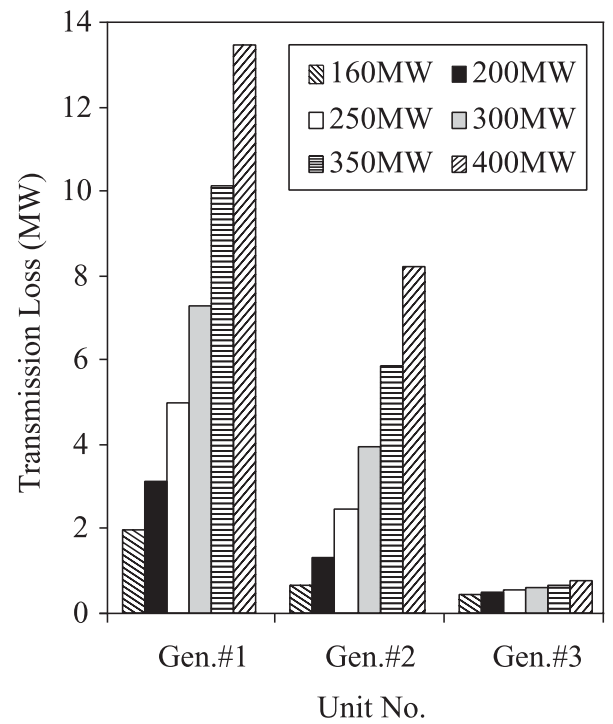

Fig. 4. Allocated loss to units at various load levels

\section{Loss Allocation for 6-bus System}

Allocated losses to three generators and total losses (MW) are shown in Table II. Here 
Table II. Allocated loss to units at several load levels

\begin{tabular}{c|c|c|c|c|c|c}
\hline Units & $160 \mathrm{MW}$ & 200MW & 250MW & 300MW & 350MW & $400 \mathrm{MW}$ \\
\hline 1 & 1.9493 & 3.1018 & 4.9565 & 7.2891 & 10.1196 & 13.4694 \\
2 & 0.6729 & 1.319 & 2.4448 & 3.9484 & 5.8571 & 8.2005 \\
3 & 0.4295 & 0.4777 & 0.5430 & 0.6118 & 0.6831 & 0.7567 \\
Total Loss & 3.0517 & 4.8985 & 7.9443 & 11.8493 & 16.6598 & 22.4266 \\
\hline
\end{tabular}

specified load levels are 200 MW, 250 MW and so on. Total loss has been calculated by DC-OPF (100MVA base). The total losses in Table III at load levels 150 MW through 250 MW are higher than those of in Table II where output of generator 3 remains lower than the bilateral power. So, the power flows toward the node 5 from other generators. For that reason, power-flow in other lines becomes large which result the larger total loss. On the other hand, the total loss in table III at load levels $300 \mathrm{MW}$ through $400 \mathrm{MW}$ are lower than those of in Table II which is logical because bilateral generator is giving fixed power. Allocated loss (without bilateral contracts) in Table III has been calculated when all generators were free to meet the demand but the load pattern was the same as that of in Table II (with bilateral contracts). Here, at load level $250 \mathrm{MW}$ allocated losses to the respective generators, (Table II and Table III), are approximately same. Generated powers of the corresponding units were also approximately same. The total values of allocated losses in Table II and Table III are the same as the total losses calculated by DCOPF respectively.

\section{Loss allocation for IEEE-118-bus system}

To show the effectiveness of the procedure, we have applied the procedure to the IEEE118-bus system. Table IV shows the allocated losses to the generator buses (PV buses) at several load levels. Total loss has been allocated to the generator buses. Using this procedure the losses can also be allocated to the demand buses.

The proposed procedure can yield negative allocations to reward generators or loads that are strategically well positioned in the network. These negative loss allocations for market purposes can be interpreted as a source of cross-subsidies (Conejo, et al. (2003). Some buses contain both generator

Table III. Allocated loss (without bilateral contracts)

\begin{tabular}{c|c|c|c|c|c|c}
\hline Units & $150 \mathrm{MW}$ & 200MW & 250MW & 300MW & $350 \mathrm{MW}$ & $400 \mathrm{MW}$ \\
\hline 1 & 2.1492 & 3.6922 & 5.0639 & 6.6085 & 8.3988 & 10.9077 \\
2 & 0.7672 & 1.6558 & 2.5106 & 3.5098 & 4.7034 & 6.4216 \\
3 & 0.1868 & 0.2258 & 0.4959 & 0.9216 & 1.487 & 1.9055 \\
Total Loss & 3.1032 & 5.5738 & 8.0704 & 11.0399 & 14.5892 & 19.2348 \\
\hline
\end{tabular}


Table IV. Allocated losses for IEEE-118-bus system (without bilateral contract)

\begin{tabular}{c|c|c|c|c|c|c|c}
\hline Unit no. & Bus & 2000MW & 2500MW & $3000 \mathrm{MW}$ & $3500 \mathrm{MW}$ & $4000 \mathrm{MW}$ & $4500 \mathrm{MW}$ \\
\hline 1 & 10 & 0.0412 & -0.0066 & -0.0697 & -0.1439 & -0.0731 & 0.0812 \\
2 & 11 & 0.0759 & -0.0377 & -0.1658 & -0.2932 & -0.2249 & 0.1424 \\
3 & 12 & 0.0060 & -0.0077 & -0.0144 & -0.0100 & $\ldots$. & $\ldots$. \\
4 & 25 & 0.0850 & 0.0236 & -0.0603 & -0.1604 & -0.0656 & 0.0106 \\
5 & 26 & 0.0215 & 0.0050 & -0.0175 & -0.0443 & -0.018 & 0.0032 \\
6 & 49 & 0.7785 & 0.9066 & 0.9969 & 1.0578 & 2.5064 & 4.6318 \\
7 & 59 & 0.0387 & 0.0323 & 0.0183 & $\ldots$. & $\ldots$. & $\ldots$. \\
8 & 61 & 0.1045 & 0.1415 & 0.1783 & 0.2142 & 0.2368 & 0.2458 \\
9 & 65 & 0.2906 & 0.6957 & 1.2818 & 2.0295 & 2.2233 & 2.2797 \\
10 & 66 & 0.2202 & 0.5827 & 1.1175 & 1.8361 & 2.1073 & 2.1377 \\
11 & 69 & 0.4086 & 0.9193 & 1.6629 & 2.6571 & 2.9300 & 2.8806 \\
12 & 80 & 0.3107 & 0.7603 & 1.4453 & 2.3888 & 2.5154 & 2.3919 \\
13 & 87 & 0.0006 & 0.0019 & 0.0036 & 0.0057 & 0.0023 & 0.0020 \\
14 & 89 & 0.7196 & 1.5248 & 2.7292 & 4.3531 & 4.3662 & 4.9626 \\
15 & 100 & 0.0199 & 0.0267 & 0.0321 & 0.0353 & 0.0239 & 0.0170 \\
16 & 103 & 0.0135 & 0.0145 & 0.0132 & 0.0093 & 0.0014 & $\ldots$. \\
17 & 111 & 0.0281 & 0.0318 & 0.0349 & 0.0373 & 0.0111 & -0.0001 \\
\hline \multicolumn{2}{l|}{ Total Loss } & 3.1631 & 5.6147 & 9.1864 & 13.9726 & 16.5424 & 19.7864 \\
\hline
\end{tabular}

and load. As a result, depending on load level, these may be considered either generator buses or load buses. More specifically, if the positive power injection from generator is greater than the load of that bus, the bus must be considered as a PV bus. If the positive power injection from generator is less than the load of that bus, the bus must be considered as a PQ bus. For example, the 36 generators in the 118-bus system connected to 20 nodes. But, out of them 17 nodes remain as PV nodes and rest 3 nodes become PQ nodes at minimum to maximum load levels allowed by the system. But, at 3500 MW load level,
16 nodes remain as PV nodes, because the node \#59 becomes PQ node. Similarly, at 4500 MW load level, the nodes \#12, \#59 and \#103 become PQ nodes. Therefore, our method takes care for the transition of PV node to PQ node and vice versa. To reward the geographically well-positioned buses, this procedure as like as other methods (Conejo, et al. 2002) can allocate negative losses. For example, negative loss allocation occurs to buses \#10, \#11, \#12, \#25 and \#26 at load level 3000 MW. But, at load level 4500 MW, negative loss occurs to bus \#111 only. The proposed method does not allocate loss 
to the bus that has neither load nor generator. This is the most important feature to avoid injustice in loss allocations.

At each load level, to calculate the preliminary losses, the ITLs are multiplied by the output of the respective generators. This preliminary loss calculation is free from the affect of ITLs in other load levels.

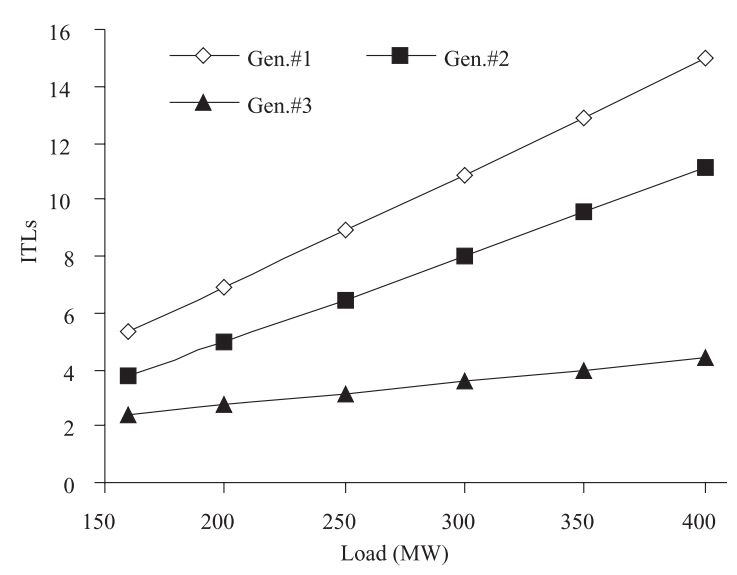

Fig. 5. ITL versus load lines

The ITLs in a specific load level do not dependent on those in other load levels. Therefore, loss allocation depending on them becomes logical.

\section{Consideration}

As the transmission line parameters are constants, in Eq. (2) the term ${ }_{\left\lceil\partial \mathrm{L} / \partial \mathrm{P}_{n}\right\rceil}$ varies with respect to node voltage angle only, so does the term $\left[\partial \mathrm{L} / \partial \mathrm{P}_{\mathrm{n}}\right]$ in Eq. (3). The difference in angles in $\sin \left(\theta_{n}-\theta_{k}\right)$ is small. It is found that for a small angle variation around zero the sine term varies linearly with respect to angle. Using the Eq. (3) in the 6-bus model power system, it is obtained that ITL varies linearly with respect to load levels (Fig. 5). It may be mentioned that, in a system where some units move from lower or upper limit to within their limits or vise versa then the slope of the ITL versus load lines become different in various load levels. So, instantaneous ITL values have been used for loss allocation.

\section{Conclusion}

In this paper, the nontrivial issue of how power losses should be allocated among generators of the transmission service has been presented. Here, we have made an important consideration on transmission loss allocation by means of ITLs among the generators including bilateral one in a deregulated power industry. To overcome the non-linear effect of power flow and power loss, we have allocated the losses according to instantaneous 'Loss Allocation Rates' $R_{n}$ and $R_{i}$ as obtained by Eq. (6) and Eq. (7) at a specific load level. Various simulation results have confirmed the effectiveness of our method. We think, the proposed loss allocation method considering bilateral contracts will be useful for independent power producers in the deregulated power industry.

\section{References}

Chiba, Y. Li., X. Ueda, T. Yamashiro, S. (2003) A method for transmission loss allocation using optimal power flow, The paper of joint 
technical meeting on Power Engineering and Power System Engineering, IEE Japan, PE-03-81.

Conejo, A.J. Arroyo, J. M. Alguacil, N. and Guijarro, A. L. (2002) Transmission loss allocation: a comparison of different practical algorithms, IEEE Trans. Power Syst., 17(3) : 571-576.

Conejo, A.J. Galiana, F.D. Arroyo, J. M. GarciaBertrand, R. Chua, C. W. and Huneault, M. (2003), Economic inefficiencies and crosssubsidies in an auction-based electricity pool, IEEE trans. on power syst., 18(1) : 211-228.

Elgerd, O.I. (1982) Electrical Energy Systems Theory: An Introduction: McGraw Hill, ch. 8.
Galiana, F.D. Phelan, M. (2000) Allocation of transmission losses to bilateral contracts in a competitive environment, IEEE transactions on power systems, 15(1).

Willis, L. Finney, J. and Ramon, G. (1996) Computing the cost of unbundled services, IEEE Computer applications in Power, 16-21.

Yamashiro, S. (1977), Optimization of power flow by DC method, The journal of the IEEJ, 97B(11) : 679-684.

Received : March 03, 2007;

Accepted : August 15, 2007 